\title{
Nanoscale
}

\section{Chiral CdSe Nanoplatelets as an Ultrasensitive Probe for Lead lon Sensing}

Received 00th January 20xx, Accepted 00th January 20xx

DOI: $10.1039 / \times 0 \times x 00000 x$

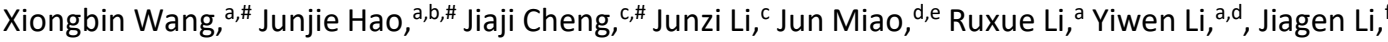
Yuhui Liu, ${ }^{a}$ Xi Zhu, ${ }^{f}$ Yanjun Liu, ${ }^{a}$ Xiao Wei Sun, ${ }^{a}$ Zikang Tang, ${ }^{d}$ Marie-Hélène Delville, ${ }^{b,}{ }^{*}$ Tingchao He, ${ }^{c,}{ }^{*}$ and Rui Chen ${ }^{\mathrm{a},}{ }^{*}$
\end{abstract}

\begin{abstract}
As opposed to traditional photoluminescence and ultra-violet based optical sensing, we present here a sensing system based on resolved optically active polarization with promising applications. It is based on the of ultrathin CdSe nanoplatelets (NPLS) when modified with either L or D -cysteine molecules (L/D-cys) as bio-to-nano ligands. The chiral ligand transfers its chiroptical activity to the achiral nanoplatelets with an anisotropy factor $\sim 10^{-4}$, which unlocks the chiral exitonic transitions and allows lead ions detection with a limit of detection (LOD) as low as $4.9 \mathrm{nM}$. Simulations and modelling based on timedependent density function theory (TD-DFT) reveal the chiral mechanism of L/D-cys capped CdSe NPLs. The presented CDbased sensing system illustrates an alternative possibility of using chiral CdSe NPLs as competitive chiral sensors for heavy metal ion detection.
\end{abstract}

\section{Introduction}

Colloidal semiconductor nanoparticles (NPs) with chirality have attracted tremendous interest due to their unique electronic and optical properties, which open the floodgate for novel sensitive chiroptical platforms as compared to their pristine achiral counterparts. ${ }^{1-4}$ Generally, the induction of chirality in semiconductor NPs can be classified as: (i) NPs with intrinsically chiral dislocations and defects, ${ }^{5-8}$ (ii) chiral surfaces formed by the chiral ligands or chiral interactions between chiral ligands and achiral semiconductor core, ${ }^{9-12}$ and (iii) chiral assemblies consisting in achiral nanocrystals. ${ }^{13-18}$ For instance, G. Markovich's group has accomplished enantioselective synthesis of intrinsically chiral mercury sulfide NPs. ${ }^{[8]}$ More recently, N. A. Kotov et al., ${ }^{19}$ reported the preparation of chiral tungsten oxide hydrate NPs with chirality transferred from the chiral ligands proline (Pro) and aspartic acid (Asp) to the tungsten oxide NPs via bio-to-nano chirality transfer. Such NPs can facilitate the formation of peptide bonds, leading to Asp-Asp and Asp-Pro dipeptides. Moreover, M. Liu and his colleague reported the synthesis of chiral assembled perovskite nanostructures through a supramolecular self-assembly approach and the

${ }^{a}$ Department of Electrical and Electronic Engineering, Southern University of Science and Technology, Shenzhen, Guangdong, 518055, P. R. China

${ }^{b}$ University of Bordeaux, CNRS, ICMCB, UMR 5026, F-33608, Pessac, France

${ }^{c}$ College of Physics and Energy, Shenzhen University, Shenzhen, Guangdong, 518060, P. R. China

${ }^{d}$ The Institute of Applied Physics and Materials Engineering, University of Macau, Avenida da Universidade, Taipa, Macau

${ }^{e}$ Department of Materials Science and Engineering, Southern University of Science and Technology, Shenzhen, Guangdong, 518055, P. R. China

fSchool of Science and Engineering, The Chinese University of Hong Kong, Guangdong, 518172, P. R. China

\# These authors contributed equally to this work perovskite NPs with circularly polarized luminescence (CPL) were successfully endowed. However, semiconductor NPs with intrinsic chirality or assembled chiral geometries often suffer from chemical laborious procedures or sophisticated fabrication processes, leading to large scale, high cost and timeconsuming production. Currently, the most universal method is the chirality transfer which is based on the electronic interactions between chiral molecules and the achiral semiconductor core. As a result, the intriguing circular dichroism (CD) peaks occur in the vicinity of the absorption bands of the colloidal NPs. ${ }^{21}$ Recently, chiral ligand capped semiconductor nanodots, ${ }^{22-25}$ nanorods ${ }^{10,26}$ and NPLs ${ }^{27-30}$ as well as their core-shell nanostructures have been extensively studied. Like in the case of metallic plasmonic NPs such as Au or $\mathrm{Ag}$, the strong coupling between chiral ligand and achiral semiconductor can induce subsequent chirality transfer from molecules to semiconductor core with an anisotropic factor close to $10^{-4}$.2,4,31-33 Such an emerging property can easily be tuned by a simple control of the size, composition and shapes of the semiconductor NPs. Therefore, it could provide great potential for applications in chiral catalysis, ${ }^{19,34}$ stereoselective synthesis, ${ }^{8,35}$ and more importantly, in chiral sensing. ${ }^{36-40}$

Heavy metal ions spread over in water sources do significantly affect human health and global environments. It is necessary to develop reliable and low-cost methods with high sensitivity for their detection as well as remediation. The most utilized approach for heavy metal ions detection up-to-now has been fluorescence-based color probe, during which semiconductor NPs would undergo emission quenching with the introduction of heavy metal ions. ${ }^{41-47}$ These ions exfoliate capping ligands around the semiconductor NPs interact with their surface and generate a photoluminescence "on-off "sensing detectable by a colorimetric method. However, such a fluorescence detection 
strategy suffers from limitations such as the lack of specificity of the fluorescent carrier materials and their relatively low sensitivity in water. However, using the emerging chirality in semiconductor NPs induced by capping ligands could be a suitable alternative for a fast and accurate heavy metal ion sensing thanks to the follow-up of the chiral response of the NPs while interacting with the targeted ions. The detection limits and sensitivity are then sensed via $C D$ spectra since $C D$ signals are quenched with increasing metal ions concentration. In addition, compared to conventional CdSe quantum dots or quantum rods, 2D CdSe NPLs with few layers exhibit unique absorption and emission properties, including extremely narrow and separated absorption peaks resulting from heavy and light hole excitations.

Herein, we synthesized cysteine-capped four layers CdSe NPLs via proper ligand exchange reaction between oleate anion and chiral cysteine molecules. The chirality transfer was monitored via electronic $C D$ measurements and the chiral genesis was investigated through theoretical modelling based on the density functional theory (DFT). Finally, the as-synthesized chiral CdSe NPLs were applied as a chiral probe to detect lead ion, and it shows high sensitivity with a LOD close to $4.9 \mathrm{nM}$. The strong interaction between the thiol groups and lead ions results in a high selectivity for chiral sensing among seven kinds of metal ions. The linear relationship between the CD intensity, the gfactor and the ion concentrations demonstrates an

a

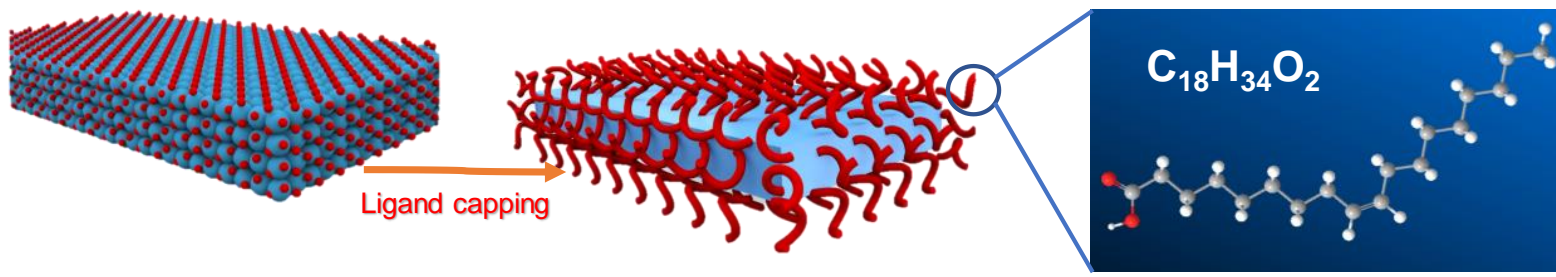

b

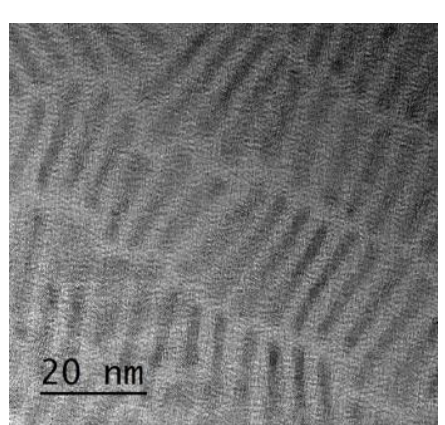

ultrasensitive lead ion detection based on such a system. These results facilitate scalable chiroptical semiconductor nanostructures toward potential applications in chiral sensing, chiral separations and design of chiral devices.

\section{Results and discussion}

First, the zinc-blende (ZB) CdSe two-dimension (2D) NPLs were synthesized directly from cadmium myristate, during which oleic acid (OA) was used as the capping agent to avoid aggregation. The CdSe NPLs with few atomic layers were prepared undergoing precursor self-assembling followed with successive purification processes (Fig. 1). The transmission electron microscope (TEM) image (Fig. 1b) presents the 2D morphology of NPLs with an average size of $5.1 \pm 1.4 \mathrm{~nm}$ in width and $25.1 \pm 2.7 \mathrm{~nm}$ in length, (Fig. S1). Fig. 1c shows the highresolution TEM image, where the $3.23 \AA$ lattice spacing corresponds to the (111) adjacent plane for ZB crystal structure can be clearly seen. The powder X-ray diffraction (XRD) patterns also indicate that the CdSe NPLs are in ZB phase. Fig. $1 \mathrm{~d}$ shows the sharp reflection at $2 \theta=25^{\circ}, 42^{\circ}$ and $49^{\circ}$, which can be assign to the (111), (220) and (311) planes of ZB phase, respectively. The results are consistent with previous reports, which demonstrate the appropriate growth CdSe NPLs. ${ }^{48}$
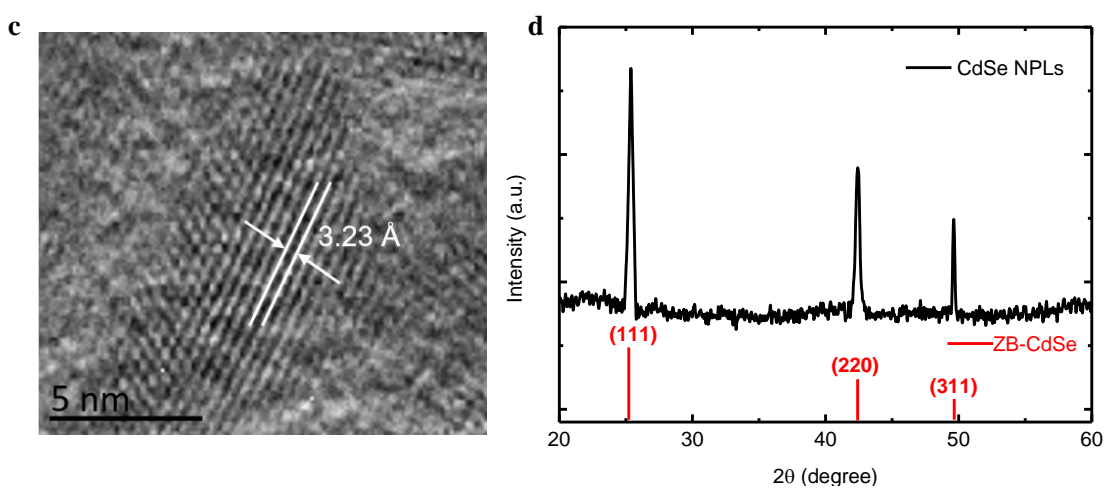

Fig. 1. (a) Schematic of ligand capping of CdSe NPLs with oleic acid. (b) TEM and (c) HRTEM image of OA capped CdSe NPLs. (d) XRD spe ctra of CdSe NPLs.

The most intriguing properties for 2D NPLs could be attributed to their unique optical response. The absorption spectrum of CdSe NPLs capped with OA (Fig. 2d) exhibits two sharp excitonic peaks located in 484 and $513 \mathrm{~nm}$. These two excitonic transitions are induced by different recombination, namely the light hole-electron for the higher energy, and the heavy holeelectron one for the lower energy absorption. The full widths at half maximum (FWHM) are 14 and $9 \mathrm{~nm}$ for light and heavy hole bands, respectively. The extremely narrow peaks are attributed to the strong quantum confinement effect in ultrathin 2D nanostructures. The observed absorption peaks indicate the thickness of NPLs herein is around four monolayers according to previous studies. ${ }^{27,49}$

After the preparation and characterization of OA-capped NPLs, the subsequent ligand exchange reaction was performed using either the L- or D-form of the chiral cysteine (Fig. S2). Due to the thiol groups in cysteine molecules, a strong $\mathrm{Cd}-\mathrm{S}$ bond is formed 
and replaces the pre-existing interaction of the CdSe surface with OA molecules (Fig. 2a-2c). After the ligand exchange, a redshift in the absorption spectrum for both light and heavy holeelectron transitions is observed to 498 and $528 \mathrm{~nm}$ respectively. The red-shift originates from the partial delocalization of the carrier wave function from the NPLs to the ligands. ${ }^{27}$ Besides, the evident broadening of the two excitonic peaks demonstrates the change of ligand density, as well as the dipole-dipole coupling after ligand exchange and the new aqueous dispersion.

Electronic CD measurements were then performed to investigate the chiroptical response of the cysteine functionalized CdSe NPLs. Indeed, bipinnate appearance of CD signals with multiple peaks arises in the vicinity of the exciton absorption of the NPLs, and a nearly mirrored CD line-shape is recorded when L- and D- cys are used as surface ligands respectively (Fig. 2e, black and red lines). Whereas in contrast, when achiral ligand (OA) or equal amount of each enantiomer of cysteine (enantiomer excess, ee $=0$ ) is used, the CD observations exhibit weak or even silent responses (pink and blue lines). All these results indicate that the induced chiroptical properties are mainly attributed to the chirality transfer from the chiral surface ligand to the achiral semiconductor core. Specifically, at the band-edge position close to $520 \mathrm{~nm}$, the obtained $C D$ shows the strongest value of the signal with an anisotropy factor close to $3 \times 10^{-4}$, which is in good agreement with previous studies. 6,10
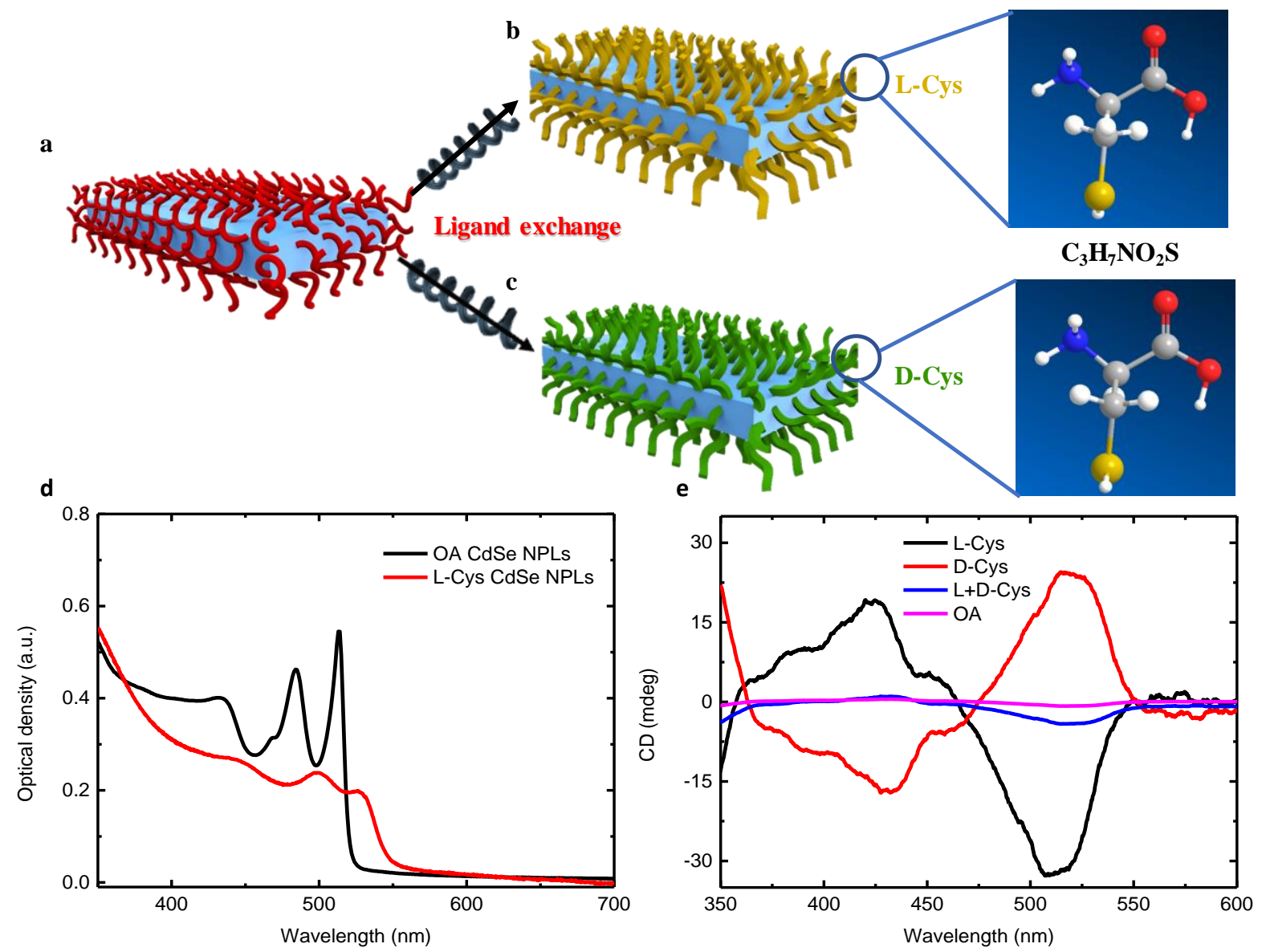

Fig. 2. (a-c) Ligand exchange process for phase transformation from oil to water with cysteine ligand replacement. (d) UV-Vis. absorption spectra of CdSe NPLs before and after ligand exchange. (e) Chirality performance using electronic CD measurements for OA, $L-$ and $D-c y s$ as well as $L+D$ (ee $=0$ ) mixed capped NPLs.

To clarify the $C D$ line shape and the chirality transfer mechanisms in these NPLs nanostructures, simulations about the molecular structure and orbital wave function in hybrid systems based on TD-DFT method were performed. The ground state geometries of L-cys and D-cys capped CdSe nanoclusters are shown in Fig. S3. Fig. $3 a$ and $3 b$ illustrate the optimized ground state geometries of L-cys and D-cys capped CdSe nanoclusters, which were utilized as the prototypes with much smaller size than in reality. All the calculations were conducted in red hat linux system. We applied four ligands to incorporate to the bare CdSe nanocluster with ZB crystal structure. For the chiral activity transfer mechanism, the surface chiral ligand would hybridize with the electronic state of achiral inorganic nanoparticle core (the excitonic band in this system), leading to the strong wave function overlap in orbital coupling. The calculated frontier molecular orbitals hybridized in the lowest unoccupied molecular orbitals (LUMO) and highest occupied molecular orbitals (HOMO) for L-cys capped CdSe nanocluster are shown in Fig. $3 \mathrm{c}$ and $3 \mathrm{~d}$. The HOMO showing strong wave function overlaps between ZB CdSe nanocluster and thiol group in chiral ligand could be clearly observed. The results demonstrate the delocalized orbitals in CdSe and cysteine in the 
organic ligand capped complexes. In contrast, the LUMO exhibits missing overlaps of the wave function of nanocluster and chiral ligands. Fig. $\mathrm{S} 4$ shows similar electronic state coupling tendency for the HOMO-1 to HOMO-3 and LUMO+1 to LUMO+3. These observations are consistent with previous simulation results for CdSe quantum dots and quantum rods. ${ }^{10,25}$ Therefore, the intrinsic chirality of bare nanoclusters were ruled out. The induction of chirality onto the achiral CdSe nanocluster could then be ascribed to the orbital coupling effect.
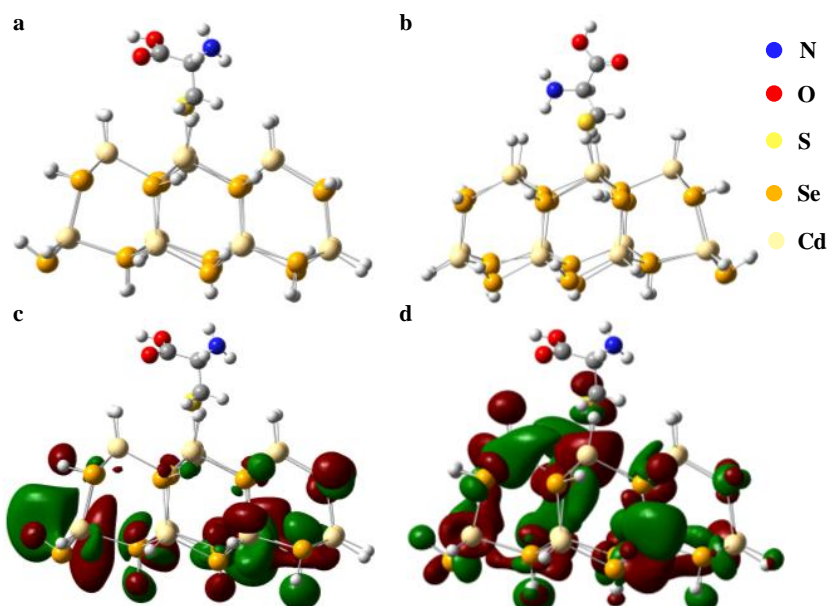

Fig 3. (a) L- (b) D-cys capped CdSe nanocluster with optimized geometries. (c) Fig 3. (a) Lulated frontier molecular orbitals of LUMO and (d) HOMO for L-cys capped CdSe nanocluster.

Based on the discussions above, it is noted that the chirality of NPLs is due to the introduction of chiral ligand. The affinity of lead for sulfur is such, that once the external lead ions are introduced, the $\mathrm{Pb}-\mathrm{S}$ bonding forms reducing the amount of chiral cysteine linked to the CdSe NPLs surface and inducing a quenching of the CD signal. The higher concentration of lead ions then results in a larger chiral signal quenching through weakening the electron state interaction between cysteine and CdSe NPLs. This provides a possible prerequisite for construction of highly sensitive heavy metal ion sensing. In this work, we utilized the lead (II) phosphate, $\mathrm{Pb}_{3}\left(\mathrm{PO}_{4}\right)_{2}$, as the lead source for detection. All the aqueous analyte samples were adjusted into $\mathrm{pH}=10$ environment. Fig. 4 a exhibits the $\mathrm{CD}$ peak response after adding various concentrations of lead ions. Their chiroptical activity showed significant decrease with increasing lead ion concentrations. The CD intensity around $520 \mathrm{~nm}$ in L- cys capped CdSe NPLs decreased from 20 mdeg to less than 1 mdeg under lead ions concentration between 0.01 to $1 \mu \mathrm{M}$. Similarly, the D-cys capped NPLs shows a signal decrease from 27 mdeg to less than 1 mdeg with the same ion concentration procedures. The UV-vis. absorption spectra in the meantime only exhibit a slight variation of the absorption intensity with the same ion concentration inductions (Fig. 4b). Fig. 4c reveals that chiral NPLs exhibit a clear linear relationship of CD intensity response at $520 \mathrm{~nm}$ versus logarithmic lead ion concentration in the range of 0.01 to $1 \mu \mathrm{M}$, with correlation coefficients of 0.998 and 0.994 for L- and D-cys capped NPLs, respectively. The LOD for lead ion sensing is determined to be $4.9 \pm 0.3 \mathrm{nM}$, from the straight line obtained in Fig. 4c.

To probe the specificity of the lead ion detection, we prepared a large enough quantity of NPLs starting material in one single batch to be able to also test six other metal ions at a $0.1 \mu \mathrm{M}$ concentration for a comparison purpose. As shown in Fig. 4d, compared to the control sample (CD intensity at around $520 \mathrm{~nm}$ (L-cys capped NPLs) 33 mdeg), the addition of $\mathrm{Ca}^{2+}, \mathrm{Mg}^{2+}, \mathrm{Fe}^{3+}$, $\mathrm{Fe}^{2+}$ resulted in the decrease of $\mathrm{CD}$ intensity in different extents in a range less than $15 \%$ of the control. The L-cys capped NPLS solutions seemed to be a bit more sensitive to the addition of $\mathrm{Cu}^{2+}$ and $\mathrm{Zn}^{2+}$ with respective decreases of 18 and $25 \%$ of $\mathrm{CD}$ signals compared to ion free control sample. Remarkably, among all the ions, the lead ions lead to the strongest CD signal quenching (58\%), of intensity of control sample. A summary of the relationship between $C D$ intensity versus ions concentrations for the seven kinds of metal ions are shown in Fig. 5. The expected linear relation significant of an effective sensing ability is present only in the case of lead ions detection. The slopes of fitted curves are summarized in Table S1. These evaluations indicate that the CdSe NPLs capped with chiral ligand are appropriate only for lead ions detection with both specificity and selectivity. As summarized in Table 1, our chiral detected performance was comparable (or even better) to the conventional optical heavy or not metal ions sensor based on photoluminescence methods. The CdSe NPLs could not be used for detecting lead ions through photoluminescence methods due to the non-luminous behavior after ligand exchange. However, the ion detection process could be realized through $C D$ spectrum detection. Our work then provides a new and simple strategy for these non-luminous semiconductors in the field of heavy metal ion detection. 


\section{Nanoscale}

\section{PAPER}
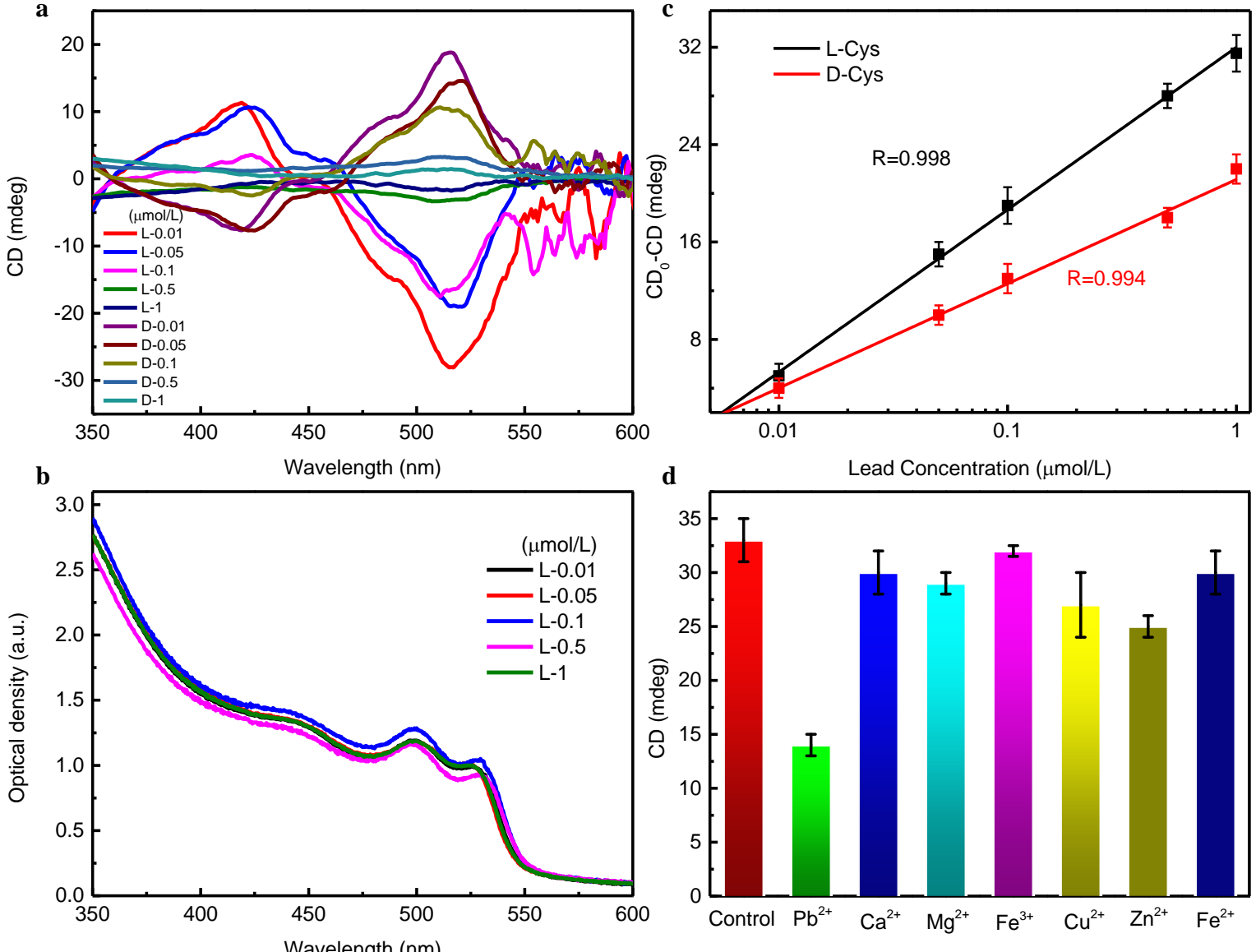

Fig. 4. (a) CD and (b) absorption measurements for lead ions detection ranging from 0.01 to $1 \mu \mathrm{M}$, both for L- and D-cys capped NPLs. (c) linear relationship between the concentrations of all metal ions are settled in $0.1 \mu \mathrm{M}$. Statistics were performed on at least 5 times for the control at an OD of 12 and 3 times for each ion.

\section{CONCLUSIONS}

In summary, ultrathin CdSe NPLs with four monolayers were prepared in this work. The ligand exchange treatment was conducted to achieve the aqueous cysteine capped NPLs. The chiral ligand has induced the chiroptical activity unlocking the exitonic transition peaks of CdSe NPLs. Moreover, we have discussed the chiral signal originated from ligand transfer mechanism according to orbital coupling simulation based on TD-DFT calculation. Taking advantage of this mechanism, highly sensitive chiroptical sensing for lead ions detection with high selectivity was demonstrated. These results revealed that such chiral super-nanostructures may serve as a versatile platform for heavy metal ions detection in waste water process.

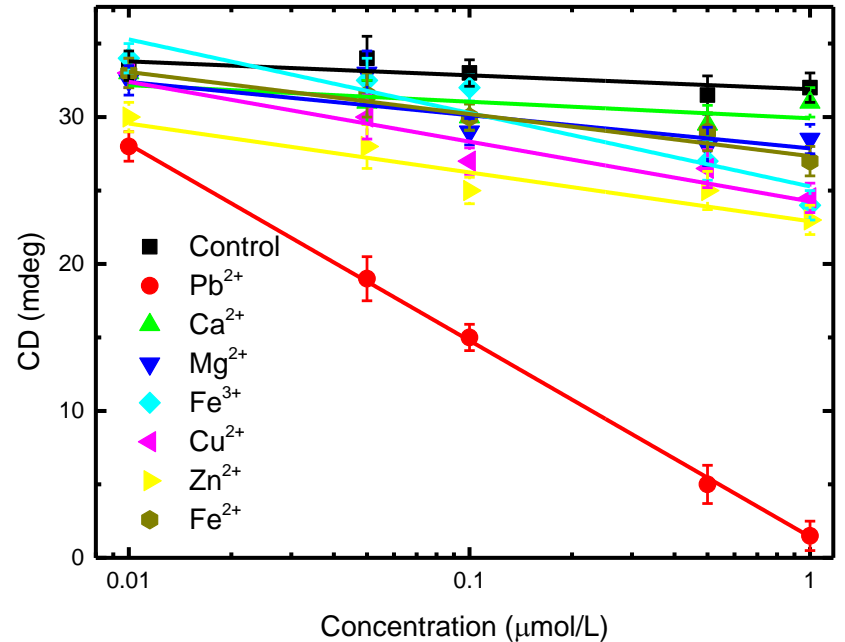

Fig. 5. Relationship between $C D$ intensity and ion concentrations for the seven kinds of metal ions. 


\section{Conclusions}

In conclusion, optical characteristics of $\mathrm{CdSe} / \mathrm{CdS} / \mathrm{ZnS}$ QDs were investigated through a comprehensive steady-state spectroscopic study. By analyzing the influence of $\mathrm{ZnS}$ shell on CdSe/CdS QDs, photostable and temperature-insensitive QDs have been obtained. Thanks to the improved optical properties of QDs, laser action is successfully obtained based on whispering gallery mode (WGM) at room temperature by using QDs as optical gain media. Furthermore, lasing at higher temperatures up to $312.6 \mathrm{~K}$ was demonstrated, which results from the superior photostability of the samples. Our result shows that CdSe/CdS/ZnS QDs have great potential as a laser material for better temperature stability and higher temperature operation.

Table 1. Lead ions limit of detection (LOD) and linear range.

\begin{tabular}{|c|c|c|c|c|}
\hline Sensing system & $\begin{array}{l}\text { Detected } \\
\text { metal ions }\end{array}$ & Range(M) (Pb) & $\begin{array}{c}\operatorname{LOD}(\mathrm{M}) \\
(\mathrm{Pb})\end{array}$ & Ref. \\
\hline $\begin{array}{c}\mathrm{Au} \\
\text { nanoparticles }\end{array}$ & $\begin{array}{l}\mathrm{Pb}, \mathrm{Fe}, \mathrm{Mn} \\
\text { etc. }\end{array}$ & $1.0 \times 10^{-7}-1.0 \times 10^{-5}$ & $1.0 \times 10^{-7}$ & {$[50]$} \\
\hline CdTe-TGA QDs & $\mathrm{Pb}$ & $2.0 \times 10^{-6}-1.0 \times 10^{-4}$ & $2.7 \times 10^{-7}$ & {$[46]$} \\
\hline Graphene oxide & $\mathrm{Pb}, \mathrm{K}$, Cu etc. & $5.0 \times 10^{-9}-3.0 \times 10^{-7}$ & $3.0 \times 10^{-9}$ & {$[51]$} \\
\hline $\begin{array}{c}\text { CdZnSe-GSH } \\
\text { QDs }\end{array}$ & $\mathrm{Pb}, \mathrm{Zn}, \mathrm{Co}$ etc. & $5.0 \times 10^{-9}-2.0 \times 10^{-5}$ & $2.7 \times 10^{-7}$ & {$[47]$} \\
\hline CdSe-DAB QDs & $\begin{array}{l}\mathrm{Cd}, \mathrm{Pb}, \mathrm{Mg} \\
\text { etc. }\end{array}$ & $1.0 \times 10^{-5}-1.5 \times 10^{-4}$ & $6.0 \times 10^{-5}$ & {$[45]$} \\
\hline $\begin{array}{c}\mathrm{CdSe} / \mathrm{CdS}-\mathrm{XO} \\
\text { QDs }\end{array}$ & $\mathrm{Pb}, \mathrm{Cu}$ etc. & $5.0 \times 10^{-8}-6.0 \times 10^{-6}$ & $2.2 \times 10^{-8}$ & {$[44]$} \\
\hline CdTe-TGA QDs & $\mathrm{Pb}, \mathrm{Zn}, \mathrm{Co}$ etc. & $2.0 \times 10^{-8}-3.6 \times 10^{-6}$ & $8.0 \times 10^{-8}$ & {$[43]$} \\
\hline $\begin{array}{c}\text { GSH-Mn-ZnS } \\
\text { QDs }\end{array}$ & $\mathrm{Pb}, \mathrm{Na}, \mathrm{Cd}$ etc. & $4.8 \times 10^{-9}-4.8 \times 10^{-7}$ & $2.2 \times 10^{-9}$ & {$[42]$} \\
\hline $\begin{array}{c}\text { MPA-Mn-ZnS } \\
\text { QDs }\end{array}$ & $\begin{array}{l}\mathrm{Pb}, \mathrm{Mg}, \mathrm{Zn} \\
\text { etc. }\end{array}$ & $4.0 \times 10^{-8}-6.0 \times 10^{-6}$ & $3.7 \times 10^{-8}$ & {$[41]$} \\
\hline CdSe-Cys NPLs & $\mathrm{Pb}, \mathrm{Fe}, \mathrm{Cu}$ etc. & $1.0 \times 10^{-8}-1.0 \times 10^{-6}$ & $4.9 \times 10^{-9}$ & $\begin{array}{l}\text { This } \\
\text { work }\end{array}$ \\
\hline
\end{tabular}

*TGA: Thioglycolic acid; GSH: Glutathione; DAB: Diaminobutane-based poly(propyleneimine) dendrimers; XO: Xylenol orange; MPA: Mercaptopropionic acid; Cys: Cysteine.

\section{EXPERIMENTAL SECTION}

Materials: Cadmium nitrate tetrahydrate $\left(\mathrm{Cd}\left(\mathrm{NO}_{3}\right)_{2} \cdot 4 \mathrm{H}_{2} \mathrm{O}\right.$, $99.997 \%)$, cadmium acetate dihydrate $\left(\mathrm{Cd}(\mathrm{Ac})_{2} \cdot 2 \mathrm{H}_{2} \mathrm{O}, 98.0 \%\right)$, oleic acid (OA, 90\%), tetramethylammonium hydroxide pentahydrate $(\mathrm{TMAH}, \geqslant 97 \%)$ were purchased from SigmaAldrich. L-cysteine hydrochloride monohydrate (99\%), Dcysteine hydrochloride monohydrate $(98 \%)$, and selenium powder (Se, $\geqslant 99.999 \%$ ) were purchased from aladdin. 1octadecene (ODE, 90\%) and sodium myristate (98\%) were purchased from J \& K. Pure water was purchased from Wahaha, China. All chemicals were used as received without further purification.
Preparation of Cadmium Myristate: Cadmium myristate was prepared according to the recipe given in the literature. For a typical synthesis, $1.23 \mathrm{~g}$ of $\mathrm{Cd}\left(\mathrm{NO}_{3}\right)_{2} \cdot 4 \mathrm{H}_{2} \mathrm{O}$ was dissolved in 40 $\mathrm{mL}$ of methanol and $3.13 \mathrm{~g}$ of sodium myristate was dissolved in $250 \mathrm{~mL}$ of methanol. After complete dissolution of $\mathrm{Cd}\left(\mathrm{NO}_{3}\right)_{2} \cdot 4 \mathrm{H}_{2} \mathrm{O}$ and sodium myristate powders, solutions were mixed and stirred vigorously around $1 \mathrm{~h}$. Then, the cadmium myristate $\left.(\mathrm{Cd} \text { (myristate })_{2}\right)$ powders were precipitated by centrifugation. and re-dispersed in methanol. The washing step with methanol was performed at least three times to remove any unreacted and/or excess precursors. After successive washing steps, the precipitated part was completely dried under vacuum overnight.

Synthesis of the CdSe Core NPLs: CdSe nanoplatelets (NPLS) having four monolayers (4 MLs) were prepared according to the procedure described in the literature with little modifications. ${ }^{52}$ For a typical synthesis, $0.17 \mathrm{~g}(0.3 \mathrm{mmol})$ of $\mathrm{Cd}$ (myristate) 2 , $0.012 \mathrm{~g}(0.15 \mathrm{mmol})$ of Se and $15 \mathrm{~mL}$ of ODE were introduced into a three-neck flask. The solution was degassed at $100{ }^{\circ} \mathrm{C}$ for an hour under vacuum. After degassing step, the solution was heated to $240{ }^{\circ} \mathrm{C}$ under argon atmosphere. $0.055 \mathrm{~g}(0.2 \mathrm{mmol})$ of $\mathrm{Cd}(\mathrm{Ac})_{2} \cdot 2 \mathrm{H}_{2} \mathrm{O}$ was swiftly added to the reaction solution. After 10 min growth at $240^{\circ} \mathrm{C}, 1 \mathrm{~mL}$ of OA was injected, and the temperature of the solution was decreased to room temperature. The cadmium precursor injection temperature and the growth time were found to be important for the resulting shape and size of CdSe core NPLs.

The resulting $4 \mathrm{ML}$ CdSe NPLs are separated from other reaction products with successive purification steps. First, the resulting mixture is centrifuged at $14,500 \mathrm{rpm}$ for $10 \mathrm{~min}$, and the supernatant is removed from the centrifuge tube. The precipitate is dried under nitrogen, re-dispersed in hexane, and centrifuged again at 4,500 rpm for $5 \mathrm{~min}$. In the second step, the supernatant is separated into another centrifuge tube, and $30 \%$ ethanol is added into the supernatant solution. In the last step, after the turbid solution is centrifuged at 4,500 rpm for $5 \mathrm{~min}$, the precipitate is dissolved in hexane and filtered with a 0.20 $\mu \mathrm{m}$ filter.

Ligand Exchange of the CdSe NPLs with Chiral Cysteine Molecules: The L- and D-cysteine ligand exchange was carried out using a previously reported method.10,27 D- or L-cysteine hydrochloride monohydrate $(0.2 \mathrm{M})$ was dissolved in DI water. The $\mathrm{pH}$ of solution was adjusted to 12 by TMAH to form the cysteine solution. $2 \mathrm{~mL}$ hexane solution containing CdSe NPLs was added into $2 \mathrm{ml}$ of cysteine solution. The mixture was stirred at room temperature for $24 \mathrm{~h}$. The reaction mixture was left to stand for $1 \mathrm{~h}$ to allow the phases to separate. The bottom aqueous layer was removed with a syringe, and the Cys-CdSe NPLs were purified by precipitation with ethanol/DI water (4:1, two times). The purified Cys-NPLs were re-dispersed in DI H2O and stored at room temperature in the dark (O.D. $=0.26$ for 2 $\mathrm{mm}$ optical length after ligand exchange).

Structural and Optical Characterization: The UV/vis absorption measurements were performed using a TU-1901 double-beam UV/vis spectrophotometer (Beijing Purkine General Instrument Co. Ltd., China), and the emission spectra were recorded on a fluoroSENS spectrophotometer (Gilden Photonics). 
CD measurements: The measurements were conducted on a JASCO J-1500 CD spectrometer at a scan rate of $20 \mathrm{~nm} / \mathrm{min}$. All $C D$ experiments were carried out in Milli- $Q$ water with a quartz cuvette ( $1 \mathrm{~cm}$ path length, Hellma). TEM images were collected using a Tecnai F30 microscope working at 300 kV. Powder X-ray diffraction (XRD) was carried out by a Rigaku Smartlab system. All the samples were at concentrations leading to peak absorbance of 1.15 O.D..

Calculation of g-Factor: In designing chiral nanoparticles for application, the anisotropy or g-factor is a key parameter to determine the optical activity due to its readily availability by comparing $C D$ spectrum with absorption spectrum. The equation is as follows: $g=\theta / 3.298 \times 10^{4} \times A$, where $\theta$ is the vertical coordinate of $C D$ spectrum, and $A$ is the value in the absorption spectrum at the same concentration used to measure the CD spectrum.

Simulation method: Gaussian 09 software is used for all chemical calculations. Ground state geometries were optimized at the DFT. UV and CD spectra were calculated with TD-DFT. B3LYP and LanL2DZ basis sets were used for all the elements in the calculations. The small CdSe clusters usually are in the form of a tetrahedron; herein, we constructed a tetrahedron-like $\mathrm{Cd}_{4} \mathrm{Se}_{9}$ cluster to mimic the interactions between the chiral light and the nanoplatelets. We take the charge states of $\mathrm{Cd}$ and $\mathrm{Se}$ as $2^{+}$and $2^{-}$. All the geometries optimized with the lowest energy were convergence to make sure available model. ${ }^{53-59}$

\section{Conflicts of interest}

The authors declare no competing financial interest.

\section{Acknowledgements}

This work is supported by the National Natural Science Foundation of China (11574130), Shenzhen Science and Technology Innovation Commission (Projects Nos. KQJSCX20170726145748464, JCYJ20150930160634263, JCYJ20170302142433007, and KQTD2015071710313656).

\section{References}

1. J. Cheng, E. H. Hill, Y. Zheng, T. He and Y. Liu, Mater. Chem. Front., 2018, 2, 662.

2. W. Ma, L. G. Xu, A. F. de Moura, X. L. Wu, H. Kuang, C. L. Xu and N. A. Kotov, Chem. Rev., 2017, 117, 8041.

3. A. Ben-Moshe, B. Maoz, A. O. Govorov and G. Markovich, Chem. Soc. Rev., 2013, 42, 7028.

4. C. Noguez and I. L. Garzon, Chem. Soc. Rev., 2009, 38, 757

5. H. Y. Ye, Y. Y. Tang, P. F. Li, W. Q. Liao, J. X. Gao, X. N. Hua, H. Cai, P. P. Shi, Y. M. You and R. G. Xiong, Science, 2018, 361, 151.

6. M. V. Mukhina, V. G. Maslov, A. V. Baranov, A. V. Fedorov, A. O. Orlova, F. Purcell-Milton, J. Govan and Y. K. Gun'ko, Nano Lett., 2015, 15, 2844.

7. A. S. Baimuratov, I. D. Rukhlenko, Y. K. Gun'ko, A. V. Baranov and A. V. Fedorov, Nano Lett., 2015, 15, 1710
8. A. Ben-Moshe, A. O. Govorov and G. Markovich, Angew. Chem., Int. Ed., 2013, 52, 1275.

9. Y. W. Li, J. J. Cheng, J. G. Li, X. Zhu, T. C. He, R. Chen and Z. K. Tang, Angew. Chem., Int. Ed., 2018, 57, 10236.

10. J. J. Cheng, J. J. Hao, H. C. Liu, J. G. Li, J. Z. Li, X. Zhu, X. D. Lin, K. Wang and T. C. He, ACS Nano, 2018, 12, 5341.

11. T. Nakashima, Y. Kobayashi and T. Kawai, J. Am. Chem. Soc., 2009, 131, 10342.

12. S. D. Elliott, M. P. Moloney and Y. K. Gun'ko, Nano Lett., 2008, 8, 2452.

13. W. C. Feng, J. Y. Kim, X. Z. Wang, H. A. Calcaterra, Z. B. Qu, L. Meshi and N. A. Kotov, Science Advances, 2017, 3, e1601159.

14. J. J. Cheng, G. Le Saux, J. Gao, T. Buffeteau, Y. Battie, P. Barois, V. Ponsinet, M. H. Delville, O. Ersen, E. Pouget and R. Oda, ACS Nano, 2017, 11, 3806.

15. Y. L. Zhou, R. L. Marson, G. van Anders, J. Zhu, G. X. Ma, P. Ercius, K. Sun, B. Yeom, S. C. Glotzer and N. A. Kotov, ACS Nano, 2016, 10, 3248.

16. J. Yeom, B. Yeom, H. Chan, K. W. Smith, S. DominguezMedina, J. H. Bahng, G. P. Zhao, W. S. Chang, S. J. Chang, A. Chuvilin, D. Melnikau, A. L. Rogach, P. J. Zhang, S. Link, P. Kral and N. A. Kotov, Nat. Mater., 2015, 14, 66.

17. B. Xu, P. He, H. Liu, P. Wang, G. Zhou and X. Wang, Angew. Chem. Int. Ed. Engl., 2014, 53, 2339.

18. E. D. Sone, E. R. Zubarev and S. I. Stupp, Angew. Chem., Int. Ed., 2002, 41, 1705.

19. S. Jiang, M. Chekini, Z. B. Qu, Y. C. Wang, A. Yeltik, Y. G. Liu, A. Kotlyar, T. Y. Zhang, B. Li, H. V. Demir and N. A. Kotov, J. Am. Chem. Soc., 2017, 139, 13701.

20. Y. H. Shi, P. F. Duan, S. W. Huo, Y. G. Li and M. H. Liu, Adv. Mater., 2018, 30.

21. G. V. Naik, V. M. Shalaev and A. Boltasseva, Adv. Mater. 2013, 25, 3264

22. M. Puri and V. E. Ferry, ACS Nano, 2017, 11, 12240.

23. F. Purcell-Milton, A. K. Visheratina, V. A. Kuznetsova, A. Ryan, A. O. Orlova and Y. K. Gunko, ACS Nano, 2017, 11, 9207.

24. J. K. Choi, B. E. Haynie, U. Tohgha, L. Pap, K. W. Elliott, B. M. Leonard, S. V. Dzyuba, K. Varga, J. Kubelka and M. Balaz, ACS Nano, 2016, 10, 3809.

25. U. Tohgha, K. K. Deol, A. G. Porter, S. G. Bartko, J. K. Choi, B. M. Leonard, K. Varga, J. Kubelka, G. Muller and M. Balaz, ACS Nano, 2013, 7, 11094.

26. X. Q. Gao, X. W. Zhang, K. Deng, B. Han, L. Y. Zhao, M. H. Wu, L. Shi, J. W. Lv and Z. Y. Tang, J. Am. Chem. Soc., 2017, 139, 8734.

$27 . \quad$ G. L. Yang, M. Kazes and D. Oron, Adv. Funct. Mater., 2018, 28, 1802012.

28. T. Stauber, T. Low and G. Gomez-Santos, Phys. Rev. Lett., 2018, 120, 046801.

29. F. Purcell-Milton, R. McKenna, L. J. Brennan, C. P. Cullen, L. Guillemeney, N. V. Tepliakov, A. S. Baimuratov, I. D. Rukhlenko, T. S. Perova, G. S. Duesberg, A. V. Baranov, A. V. Fedorov and Y. K. Gun'ko, ACS Nano, 2018, 12, 954.

30. X. Gao, X. Zhang, L. Zhao, P. Huang, B. Han, J. Lv, X. Qiu, S.H. Wei and Z. Tang, Nano Lett., 2018, 18, 6665.

31. X. A. Wang and Z. Y. Tang, Small, 2017, 13.

32. S. M. Morrow, A. J. Bissette and S. P. Fletcher, Nat. Nanotechnol., 2017, 12, 410.

33. X. Lan and Q. B. Wang, Adv. Mater., 2016, 28, 10499.

34. C. Hao, L. Xu, W. Ma, X. Wu, L. Wang, H. Kuang and C. Xu, Adv. Funct. Mater., 2015, 25, 5816.

35. I. V. Martynenko, V. A. Kuznetsova, I. K. Litvinov, A. O. Orlova, V. G. Maslov, A. V. Fedorov, A. Dubavik, F. PurcellMilton, Y. K. Gun'ko and A. V. Baranov, Nanotechnology, 2016, 27, 075102.

36. M. Matuschek, D. P. Singh, H. H. Jeong, M. Nesterov, T. 
Weiss, P. Fischer, F. Neubrech and N. Liu, Small, 2018, 14, 1702990.

37. Z. Y. Bao, W. Zhang, Y. L. Zhang, J. J. He, J. Y. Dai, C. T. Yeung, G. L. Law and D. Y. Lei, Angew. Chem., Int. Ed., 2017, 56, 1283.

38. M. V. Mukhina, I. V. Korsakov, V. G. Maslov, F. Purcell-Milton J. Govan, A. V. Baranov, A. V. Fedorov and Y. K. Gun'ko, Sci. Rep., 2016, 6, 24177.

39. Y. H. Xia, Y. L. Zhou and Z. Y. Tang, Nanoscale, 2011, 3, 1374

40. K. M. Mayer and J. H. Hafner, Chem. Rev., 2011, 111, 3828.

41. T. T. Gan, N. J. Zhao, G. F. Yin, M. D. Tu, J. G. Liu and W. Q. Liu, New J. Chem., 2017, 41, 13425.

42. J. L. Chen, Y. X. Zhu and Y. Zhang, Spectroc. Acta Pt. A-Mol. Biomol. Spectr., 2016, 164, 98.

43. S. Xu, S. H. Xu, Y. S. Zhu, W. Xu, P. W. Zhou, C. Y. Zhou, B. Dong and H. W. Song, Nanoscale, 2014, 6, 12573.

44. Q. Zhao, X. L. Rong, L. Chen, H. B. Ma and G. H. Tao, Talanta, 2013, 114, 110.

45. M. Algarra, B. B. Campos, B. Alonso, M. S. Miranda, A. M. Martinez, C. M. Casado and J. C. G. E. da Silva, Talanta, 2012, 88, 403.

46. H. M. Wu, J. G. Liang and H. Y. Han, Microchim. Acta, 2008, $161,81$.

47. E. M. Ali, Y. G. Zheng, H. H. Yu and J. Y. Ying, Anal. Chem., 2007, 79, 9452.

48. Y. Kelestemur, M. Olutas, S. Delikanli, B. Guzelturk, M. Z. Akgul and H. V. Demir, J. Phys. Chem. C, 2015, 119, 2177.

49. S. Ithurria, M. D. Tessier, B. Mahler, R. P. S. M. Lobo, B. Dubertret and A. Efros, Nat. Mater., 2011, 10, 936.

50. F. Chai, C. A. Wang, T. T. Wang, L. Li and Z. M. Su, ACS Appl. Mater. Interfaces, 2010, 2, 1466.

51. Y. F. Bai, L. Zhao, Z. H. Chen, H. Y. Wang and F. Feng, Anal. Methods, 2014, 6, 8120.

52. Y. Kelestemur, B. Guzelturk, O. Erdem, M. Olutas, K. Gungor and H. V. Demir, Adv. Funct. Mater., 2016, 26, 3570.

53. A. N. Beecher, X. H. Yang, J. H. Palmer, A. L. LaGrassa, P. Juhas, S. J. L. Billinge and J. S. Owen, J. Am. Chem. Soc., 2014, 136, 10645.

54. T. Vossmeyer, G. Reck, L. Katsikas, E. T. K. Haupt, B. Schulz and $\mathrm{H}$. Weller, Science, 1995, 267, 1476.

55. C. T. Lee, W. T. Yang and R. G. Parr, Phys. Rev. B, 1988, 37, 785.

56. W. R. Wadt and P. J. Hay, J. Chem. Phys., 1985, 82, 284.

57. P. J. Hay and W. R. Wadt, J. Chem. Phys., 1985, 82, 299.

58. P. J. Hay and W. R. Wadt, J. Chem. Phys., 1985, 82, 270.

59. R. A. Gaussian09, M. J. Frisch, G. W. Trucks, H. B. Schlegel, G. E. Scuseria, M. A Robb, J. R. Cheeseman, G. Scalmani, V. Barone, G. A. Petersson, H. Nakatsuji, X. Li, M. Caricato, A. Marenich, J. Bloino, B. G. Janesko, R. Gomperts, B. Mennucci, H. P. Hratchian, J. V. Ortiz, A. F. Izmaylov, J. L. Sonnenberg, D. Williams-Young, F. Ding, F. Lipparini, F. Egidi, J. Goings, B. Peng, A. Petrone, T. Henderson, D. Ranasinghe, V. G. Zakrzewski, J. Gao, N. Rega, G. Zheng, W. Liang, M. Hada, M. Ehara, K. Toyota, R. Fukuda, J. Hasegawa, M. Ishida, T. Nakajima, Y. Honda, O. Kitao, H. Nakai, T. Vreven, K. Throssell, J. A. Montgomery, Jr., J. E. Peralta, F. Ogliaro, M. Bearpark, J. J. Heyd, E. Brothers, K. N. Kudin, V. N. Staroverov, T. Keith, R. Kobayashi, J. Normand, K. Raghavachari, A. Rendell, J. C. Burant, S. S. Iyengar, J. Tomasi, M. Cossi, J. M. Millam, M. Klene, C. Adamo, R. Cammi, J. W. Ochterski, R. L. Martin, K. Morokuma, O. Farkas, J. B. Foresman, and D. J. Fox, Gaussian, Inc., Wallingford CT, 2016. 